Journal of Applied Fluid Mechanics, Vol. 4, No. 4, pp. 77-83, 2011.

Available online at www.jafmonline.net, ISSN 1735-3572, EISSN 1735-3645.

DOI: $10.36884 / \mathrm{jafm} \cdot 4.04 .11949$

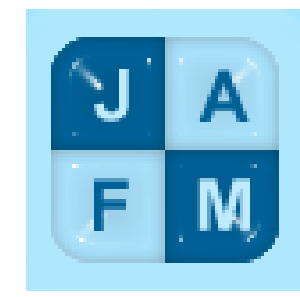

\title{
Solutions in Variably Inclined MHD Flows
}

\author{
C. S. Bagewadi ${ }^{1}$ and S. Bhagya ${ }^{2 \dagger}$ \\ ${ }^{1}$ Department of Studies in Mathematics, Kuvempu University, Jnana Sahyadri, -577 451, Shimoga, India \\ ${ }^{2}$ Department of Mathematics, P.E.S. College, Bangalore-560 050, India \\ †Corresponding Author Email: bhagyapes@yahoo.co.in
}

(Received October 8, 2009; accepted August 3, 2010)

\begin{abstract}
We study the plane MHD flows when the velocity and magnetic fields are variably inclined and investigate the steady viscous incompressible flow problems of a fluid having infinite electrical conductivity in the presence of a magnetic field. Accounting for infinite electrical conductivity makes the flow problem realistic and attractive because the magnetic Reynolds number is very small for most liquid metals. Particular problems are discussed when magnetic lines are variably inclined but nowhere aligned with streamlines, when the fluid is viscous and non-viscous. Streamlines are parabolic as shown in the graphs.
\end{abstract}

Keywords: Streamlines, Magnetic fields, Incompressible.

\section{INTRODUCTION}

A vast amount of research has been carried out on the motion of electrically conducting fluids, moving in a magnetic field. Mathematical complexity of the phenomenon induced many researchers to adopt a rather useful alternate technique of investigating special classes of flows such a aligned or parallel flows, crossed or orthogonal flows, constantly inclined flows and transverse flows. Chandna and co-workers $(1979,1982,1989,1990)$ studied finitely conducting orthogonal magneto hydrodynamic plane flows. In which they discussed that the velocity and magnetic field vectors are mutually orthogonal everywhere in the flow region. Bagewadi and Siddabasappa $(1993,1995)$ have studied Steady plane rotating MHD flows by using differential geometry technique and hodograph transformation. Bagewadi and Bhagya (2004) investigated the Behavior of streamlines in aligned flow by using differential geometry technique and hodograph transformation. Bagewadi and Bhagya (2006) obtained solutions for second grade fluid in $(\phi, \psi)$ net, where $\phi(\mathrm{x}, \mathrm{y})=$ constant, an arbitrary family of curves and $\psi(\mathrm{x}, \mathrm{y})=$ constant stream lines. The solutions for steady plane orthogonal flow of second grade fluids using complex variable techniques were carried out by Bagewadi and Bhagya (2007). Rahmati and shrafizaadeh (2009) analyzed a 19-bit Incompressible Generalized Lattice Boltzmann (IGLB) method for three-dimensional incompressible fluid flow simulation. Equilibrium moments in moment space are derived from an incompressible BGKLB method. Very recently Anjali Devi and Ganga (2010) analyzed MHD flow with heat transfer in a porous medium over a stretching porous surface with viscous and Joule dissipation effects. The present paper investigates the steady viscous incompressible flow problems of a fluid having infinite electrical conductivity in the presence of a magnetic field. Since the magnetic Reynolds number is very small for most liquid metals, our accounting for infinite electrically conductivity makes the flow problem realistic and attractive from both a mathematical and a physical point of view.

The plan of this paper is as follows; in section 2, the basic equations are formulated by using the stream function and the equations written in magnetic flux function. In section 3, Cartesian plane for inclined flows. In section 4, we discussed two problems for parallel straight streamlines and magnetic lines.

\section{BASIC EQUATIONS}

The steady plane flow of an incompressible electrically conducting viscous fluid of infinite electrical conductivity is governed by the following system of equations, Chandna and co-workers (1989)

$$
\begin{aligned}
& \operatorname{div} \mathbf{v}=0 \\
& (\mathbf{v} \cdot \operatorname{grad}) \mathbf{v}+\operatorname{gradp}=\mathrm{R}_{\mathrm{H}}(\operatorname{curl} H \times H)+\frac{1}{\mathrm{Re}} \nabla^{2} \mathbf{v} \\
& \operatorname{curl}(\mathbf{v} \times \mathbf{H})=0 \\
& \operatorname{dit} \mathbf{H}=0
\end{aligned}
$$

where $\mathbf{v}$ the velocity vector field, $\mathbf{H}$ the magnetic field vector, $\mathrm{p}$ the pressure function, Re the Reynold number and $\mathrm{R}_{\mathrm{H}}$ the magnetic pressure number. We write (2.1) to (2.4) in Cartesian co-ordinate system 
$\frac{\partial u}{\partial x}+\frac{\partial v}{\partial y}=0$

$u \frac{\partial u}{\partial x}+v \frac{\partial u}{\partial y}+\frac{\partial p}{\partial x}=\frac{1}{\operatorname{Re}}\left(\frac{\partial^{2} u}{\partial x^{2}}+\frac{\partial^{2} u}{\partial y^{2}}\right)-$

$R_{H} H_{2}\left(\frac{\partial H_{2}}{\partial x}-\frac{\partial H_{1}}{\partial y}\right)$

$u \frac{\partial v}{\partial x}+v \frac{\partial v}{\partial y}+\frac{\partial p}{\partial y}=\frac{1}{\operatorname{Re}}\left(\frac{\partial^{2} v}{\partial x^{2}}+\frac{\partial^{2} v}{\partial y^{2}}\right)+$

$R_{H} H_{1}\left(\frac{\partial H_{2}}{\partial x}-\frac{\partial H_{1}}{\partial y}\right)$

$u H_{2}-v H_{1}=k$

$\frac{\partial H_{1}}{\partial x}+\frac{\partial H_{2}}{\partial y}=0$

where $u=\frac{u^{*}}{U_{0}}, \quad v=\frac{v^{*}}{U_{0}}$ are non dimensional velocity component, $H_{1}=\frac{H_{1}^{*}}{H_{0}}, H_{2}=\frac{H_{2}^{*}}{H_{0}}$ are the non dimensional components of the magnetic field and $p=\frac{p^{*}}{\rho U_{0}^{2}}$ is the non dimensional pressure function. $\operatorname{Re}=\frac{\rho U_{0} L}{\mu}$ and $R_{H}=\frac{\mu H_{0}^{2}}{\rho U_{0}^{2}}$ are respectively the flow Reynolds number and the magnetic pressure number.

The constant $\mathrm{k}$ is an arbitrary constant and $\alpha(x, y)$ is the angle between the velocity vector $\mathbf{v}$ and the magnetic vector $\mathbf{H}$ at any point $(\mathrm{x}, \mathrm{y})$. The constants $\mathrm{L}$, $\mathrm{U}_{0}$ and $\mathrm{H}_{0}$ are characteristic length, speed and magnetic field strength respectively.

Introducing the two-dimensional vorticity function $\omega$, the current density function $\mathrm{j}$ and energy function $\mathrm{e}$ defined by

$\omega=\frac{\partial v}{\partial x}-\frac{\partial u}{\partial y}, j=\frac{\partial H_{2}}{\partial x}-\frac{\partial H_{1}}{\partial y}, e=\frac{1}{2} q^{2}+p$

where $\mathrm{q}^{2}=\mathrm{u}^{2}+\mathrm{v}^{2}$, the above system of equations is replaced by the following system;

$\frac{\partial u}{\partial x}+\frac{\partial v}{\partial y}=0 \quad$ (Continuity)

$\frac{\partial e}{\partial x}-v \omega=-\frac{1}{\operatorname{Re}} \frac{\partial \omega}{\partial y}-R_{H} j H_{2}$

$\frac{\partial e}{\partial y}+u \omega=\frac{1}{\operatorname{Re}} \frac{\partial \omega}{\partial x}+R_{H} j H_{1}$ (linear momentum)

$u H_{2}-v H_{1}=k \quad$ (Diffusion)

$\frac{\partial H_{1}}{\partial x}+\frac{\partial H_{2}}{\partial y}=0 \quad$ (Solenoid)

$\frac{\partial v}{\partial x}-\frac{\partial u}{\partial y}=\omega \quad$ (Vorticity)

$\frac{\partial H_{2}}{\partial x}-\frac{\partial H_{1}}{\partial y}=j \quad$ (Current density) of seven equations in seven unknowns $\mathrm{u}, \mathrm{v}, \mathrm{H}_{1}, \mathrm{H}_{2}, \omega$, $\mathrm{j}$ and $\mathrm{e}$ as function of $\mathrm{x}, \mathrm{y}$. The advantage of this system over the system of Eqs. (2.5) to (2.9) is that the order of the partial differential equations has decreased from two to one. Martin (1971) has, with much success, used a similar reduction of order to study viscous nonMHD flows.

\section{INCLINED PLANE FLOWS}

We consider variably inclined plane flows and let $\alpha$ (x, $\mathrm{y})$ be the variable angle such that $\alpha(\mathrm{x}, \mathrm{y}) \neq 0$ for every $(x, y)$ in the flow region. The vector and scalar products of $\mathrm{v}$ and $\mathrm{H}$, using the diffusion equation in (2.13), yield

$$
\begin{gathered}
u H_{2}-v H_{1}=q H \sin \alpha=k \\
u H_{1}+v H_{2}=q H \cos \alpha=k \cot \alpha
\end{gathered}
$$

where $q=\sqrt{u^{2}+v^{2}}$ and $H=\sqrt{H_{1}^{2}+H_{2}^{2}}$.

Considering these as two linear algebraic equations in the unknowns $\mathrm{H}_{1}, \mathrm{H}_{2}$, we solve for $\mathrm{H}_{1}, \mathrm{H}_{2}$ in terms of $\mathrm{u}$, $\mathrm{v}$ and $\alpha$. We have

$H_{1}=\frac{k}{q^{2}}(u \cot \alpha-v), H_{2}=\frac{k}{q^{2}}(v \cot \alpha+u)$

One can eliminate $\mathrm{H}_{1}$ and $\mathrm{H}_{2}$ from the system of Eqs. (2.11) to (2.16) by using Eq. (3.2) and obtain a system to be solved for $\mathrm{u}, \mathrm{v}, \mathrm{e}, \omega, \mathrm{j}$ and $\alpha$ as functions of $\mathrm{x}, \mathrm{y}$. Eliminating $\mathrm{H}_{1}$ and $\mathrm{H}_{2}$ from the system of Eqs. (2.11) to (2.16), by using (3.2), we obtain the following system of six partial differential equations

$\frac{\partial u}{\partial x}+\frac{\partial v}{\partial y}=0$

$\frac{1}{\operatorname{Re}} \frac{\partial \omega}{\partial y}-v \omega+R_{H}\left(\frac{u+v \cot \alpha}{q^{2}}\right) j=-\frac{\partial e}{\partial x}$

$\frac{1}{\operatorname{Re}} \frac{\partial \omega}{\partial x}-v \omega+R_{H}\left(\frac{u \cot \alpha-v}{q^{2}}\right) j=-\frac{\partial e}{\partial y}$

$\frac{\partial}{\partial x}\left(\frac{u \cot \alpha-v}{q^{2}}\right)+\frac{\partial}{\partial y}\left(\frac{u+v \cot \alpha}{q^{2}}\right)=0$

$\frac{\partial v}{\partial x}-\frac{\partial u}{\partial y}=\omega$

$k \frac{\partial}{\partial x}\left(\frac{u+v \cot \alpha}{q^{2}}\right)-k \frac{\partial}{\partial y}\left(\frac{u \cot \alpha-v}{q^{2}}\right)=j$

For six unknown functions $\mathrm{u}, \mathrm{v}, \mathrm{j}, \omega, \mathrm{e}, \alpha$ of $\mathrm{x}, \mathrm{y}$. Once a solution of this system is determined, the pressure and the magnetic field are obtained by using the definition of e in (2.10) and Eq. (3.2) respectively.

The equation of continuity (3.3) implies the existence of a stream function $\psi(\mathrm{x}, \mathrm{y})$ such that $d \psi=-v d x+u d y$ 
C.S. Bagewadi and S. Bhagya / JAFM, Vol. 4, No. 4, pp. 77-83, 2011.

$$
\text { or } \quad \frac{\partial \psi}{\partial x}=-v, \quad \frac{\partial \psi}{\partial y}=u
$$

Introducing $\psi(\mathrm{x}, \mathrm{y})$ into the system of Eqs. (3.3) to (3.8), it follows that Eq.(3.3) is identically satisfied and this system may be replaced by

$$
\frac{1}{\operatorname{Re}} \frac{\partial \omega}{\partial y}+\omega \frac{\partial \psi}{\partial x}+k R_{H}\left[\frac{\frac{\partial \psi}{\partial y}-\frac{\partial \psi}{\partial x} \cot \alpha}{\left(\frac{\partial \psi}{\partial x}\right)^{2}+\left(\frac{\partial \psi}{\partial y}\right)^{2}}\right] j=\frac{-\partial e}{\partial x}
$$

$$
\frac{1}{\operatorname{Re}} \frac{\partial \omega}{\partial x}-\omega \frac{\partial \psi}{\partial y}+k R_{H}\left[\frac{\frac{\partial \psi}{\partial x}+\frac{\partial \psi}{\partial y} \cot \alpha}{\left(\frac{\partial \psi}{\partial x}\right)^{2}+\left(\frac{\partial \psi}{\partial y}\right)^{2}}\right] j=\frac{\partial e}{\partial y}
$$

$\frac{\partial}{\partial x}\left[\frac{\frac{\partial \psi}{\partial y} \cot \alpha+\frac{\partial \psi}{\partial x}}{\left(\frac{\partial \psi}{\partial x}\right)^{2}+\left(\frac{\partial \psi}{\partial y}\right)^{2}}\right]+\frac{\partial}{\partial y}\left[\frac{\frac{\partial \psi}{\partial y}-\frac{\partial \psi}{\partial x} \cot \alpha}{\left(\frac{\partial \psi}{\partial x}\right)^{2}+\left(\frac{\partial \psi}{\partial y}\right)^{2}}\right]=0$

$-\left(\frac{\partial^{2} \psi}{\partial x^{2}}+\frac{\partial^{2} \psi}{\partial y^{2}}\right)=\omega$

$k\left[\frac{\partial}{\partial x}\left(\frac{\frac{\partial \psi}{\partial y}-\frac{\partial \psi}{\partial x} \cot \alpha}{\left(\frac{\partial \psi}{\partial x}\right)^{2}+\left(\frac{\partial \psi}{\partial y}\right)^{2}}\right)-\frac{\partial}{\partial y}\left(\frac{\frac{\partial \psi}{\partial y} \cot \alpha+\frac{\partial \psi}{\partial x}}{\left(\frac{\partial \psi}{\partial x}\right)^{2}+\left(\frac{\partial \psi}{\partial y}\right)^{2}}\right)\right]=j$

We use the integrability condition $\frac{\partial^{2} e}{\partial x \partial y}=\frac{\partial^{2} e}{\partial y \partial x}$ for the integration of $\mathrm{e}(\mathrm{x}, \mathrm{y})$ from the linear momentum equations (3.10), (3.11) and use (3.12) to obtain

$$
\begin{aligned}
& \frac{1}{\operatorname{Re}}\left[\frac{\partial^{2} \omega}{\partial x^{2}}+\frac{\partial^{2} \omega}{\partial y^{2}}\right]+\left(\frac{\partial \psi}{\partial x} \frac{\partial \omega}{\partial y}-\frac{\partial \psi}{\partial y} \frac{\partial \omega}{\partial x}\right)+k R_{H} \\
& {\left[\frac{\left(\frac{\partial \psi}{\partial y}-\frac{\partial \psi}{\partial x} \cot \alpha\right) \frac{\partial j}{\partial y}+\left(\frac{\partial \psi}{\partial x}+\frac{\partial \psi}{\partial y} \cot \alpha\right) \frac{\partial j}{\partial x}}{\left(\frac{\partial \psi}{\partial x}\right)^{2}+\left(\frac{\partial \psi}{\partial y}\right)^{2}}\right]=0}
\end{aligned}
$$

Equations (3.12) and (3.15) form a system of two equations in two unknown functions $\psi(\mathrm{x}, \mathrm{y})$ and $\alpha(\mathrm{x}, \mathrm{y})$ after $\omega$ and $\mathrm{j}$ are eliminated from these equations by employing their expressions from (3.13) and (3.14). Given a solution of this system, the velocity field $\mathrm{u}, \mathrm{v}$ is given by (3.9), the vorticity $\omega$ is given by (3.13), the current density $\mathrm{j}$ is given by (3.14), the magnetic field is given by (3.2) and e is given by the integration of (3.10) and (3.11). Finally, the pressure function is determined from (2.6) and (2.7).

Taking the second approach, we consider the equation (3.1) to be our two linear equations in unknowns u, v and obtain

$$
\begin{aligned}
& u=\frac{k}{\mathbf{H}^{2}}\left(H_{1} \cot \alpha+H_{2}\right) \\
& v=\frac{k}{\mathbf{H}^{2}}\left(H_{2} \cot \alpha-H_{1}\right)
\end{aligned}
$$

We eliminate functions $\mathrm{u}$ and $\mathrm{v}$ from the system of Eqs. (2.11) to (2.16) by using Eq. (3.16) and obtain the following system of six partial differential equations

$$
\begin{aligned}
& \frac{\partial H_{1}}{\partial x}+\frac{\partial H_{2}}{\partial y}=0 \\
& \frac{1}{\operatorname{Re}} \frac{\partial \omega}{\partial y}+R_{H} j H_{2}+k\left(\frac{H_{2} \cot \alpha-H_{1}}{\mathbf{H}^{2}}\right)=-\frac{\partial e}{\partial x} \\
& \frac{1}{\operatorname{Re}} \frac{\partial \omega}{\partial x}+R_{H} j H_{1}-k\left(\frac{H_{1} \cot \alpha+H_{2}}{\mathbf{H}^{2}}\right)=\frac{\partial e}{\partial y} \\
& \frac{\partial}{\partial x}\left(\frac{k\left(H_{2} \cot \alpha-H_{1}\right)}{\mathbf{H}^{2}}\right)- \\
& \frac{\partial}{\partial y}\left(\frac{k\left(H_{1} \cot \alpha+H_{2}\right)}{\mathbf{H}^{2}}\right)=\omega \\
& \frac{\partial H_{2}}{\partial x}-\frac{\partial H_{1}}{\partial y}=j \\
& \frac{\partial}{\partial x}\left(\frac{\left(H_{1} \cot \alpha+H_{2}\right)}{\mathbf{H}^{2}}\right)- \\
& \frac{\partial}{\partial y}\left(\frac{\left(H_{2} \cot \alpha-H_{1}\right)}{\mathbf{H}^{2}}\right)=0
\end{aligned}
$$

For the six unknown functions $\mathrm{H}_{1}, \mathrm{H}_{2}, \mathrm{j}, \omega$, e and $\alpha$ of $\mathrm{x}, \mathrm{y}$. Introducing the magnetic flux function defined by

$$
\begin{aligned}
& d \phi=-H_{2} d x+H_{1} d y_{1} \\
& o r-\frac{\partial \phi}{\partial x}=H_{2}, \frac{\partial \phi}{\partial y}=H_{1}
\end{aligned}
$$

and eliminate $\mathrm{H}_{1}$ and $\mathrm{H}_{2}$. Use the integrability condition $\frac{\partial^{2} e}{\partial x \partial y}=\frac{\partial^{2} e}{\partial y \partial x}$ for the integration of $\mathrm{e}(\mathrm{x}, \mathrm{y})$ from the linear momentum equations for in viscid flows and have equations

$$
\begin{aligned}
& R_{H}\left[\frac{\partial j}{\partial x} \frac{\partial \phi}{\partial y}-\frac{\partial j}{\partial y} \frac{\partial \phi}{\partial x}\right] \\
& +\frac{k}{\left(\frac{\partial \phi}{\partial x}\right)^{2}+\left(\frac{\partial \phi}{\partial y}\right)^{2}} \\
& {\left[\left(\frac{\partial \omega}{\partial x}\right)\left(\frac{\partial \phi}{\partial x}-\frac{\partial \phi}{\partial y} \cot \alpha\right)\right.} \\
& \left.+\left(\frac{\partial \omega}{\partial y}\right)\left(\frac{\partial \phi}{\partial y}+\frac{\partial \phi}{\partial x} \cot \alpha\right)\right]=0
\end{aligned}
$$




$$
\begin{gathered}
\frac{\partial}{\partial x}\left[\frac{\frac{\partial \phi}{\partial y} \cot \alpha-\frac{\partial \phi}{\partial x}}{\left(\frac{\partial \phi}{\partial x}\right)^{2}+\left(\frac{\partial \phi}{\partial y}\right)^{2}}\right]-\frac{\partial}{\partial y}\left[\frac{\frac{\partial \phi}{\partial x} \cot \alpha+\frac{\partial \phi}{\partial y}}{\left(\frac{\partial \phi}{\partial x}\right)^{2}+\left(\frac{\partial \phi}{\partial y}\right)^{2}}\right]=0 \\
\omega=-k\left[\frac{\left.\frac{\partial}{\partial x}\left[\frac{\frac{\partial \phi}{\partial y}+\frac{\partial \phi}{\partial x} \cot \alpha}{\left(\frac{\partial \phi}{\partial x}\right)^{2}+\left(\frac{\partial \phi}{\partial y}\right)^{2}}\right]+\frac{\partial}{\partial y}\left[\frac{\frac{\partial \phi}{\partial x}+\frac{\partial \phi}{\partial y} \cot \alpha}{\left(\frac{\partial \phi}{\partial x}\right)^{2}+\left(\frac{\partial \phi}{\partial y}\right)^{2}}\right]\right)}{j=-\left(\frac{\partial^{2} \phi}{\partial x^{2}}+\frac{\partial^{2} \phi}{\partial y^{2}}\right)}\right.
\end{gathered}
$$

form a system of two equations in two unknown functions. $\phi(\mathrm{x}, \mathrm{y})$ and $\alpha(\mathrm{x}, \mathrm{y})$ after $\omega$ and $\mathrm{j}$ are eliminated from these equations by employing (3.26) and (3.27). Given a solution of this system the magnetic field is given by (3.23), the vorticity by (3.26), the current density by (3.27), the velocity field by (3.16) and $\mathrm{e}$ by the integration of the linear momentum Eqs. (3.4) and (3.5). Finally the pressure function is determined from (2.6) and (2.7).

\section{APplications}

\subsection{Problem 1}

Next we investigate that, when the magnetic lines are variably inclined but no where aligned with the streamlines. Also we discuss when the magnetic lines are constantly inclined but non-aligned with the streamlines.

We assume that

$\psi=\mathrm{G}(\mathrm{y}), \mathrm{G}(\mathrm{y}) \neq 0$

where $\mathrm{G}^{\prime}(\mathrm{y})$ is the derivative with respect to the argument. Employing this expression for $\psi$ in Eqs. (3.13) and (3.14), we get

$\omega=-G^{\prime \prime}(y), \quad j=-k \frac{\partial}{\partial y}\left(\frac{\cot \alpha}{G^{\prime}(y)}\right)$

Eliminating $\psi, \omega$ and $\mathrm{j}$ from the Eqs. (3.12) and (3.15) by using their expressions from (4.1) and (4.2), we find that $\mathrm{G}(\mathrm{y})$ and $\cot \alpha$ must satisfy

$$
\frac{\partial}{\partial x}(\cot \alpha)=\frac{G^{\prime \prime}(y)}{G^{\prime}(y)}
$$

and

$$
\begin{aligned}
& G^{\prime}(y) G^{\prime \prime}(y)+R^{2} \operatorname{Re} R_{H}\left[\frac{\partial^{2}}{\partial y^{2}}\left(\frac{\cot \alpha}{G^{\prime}(y)}\right)+\right. \\
& \left.\cot \alpha\left(\frac{\partial^{2}}{\partial x \partial y}\left(\frac{\cot \alpha}{G^{\prime}(y)}\right)\right)\right]=0
\end{aligned}
$$

Solving (4.3), we have

$$
\cot \alpha=\frac{G^{\prime \prime}(y)}{G^{\prime}(y)} x+F(y)
$$

Where $\mathrm{F}(\mathrm{y})$ is an arbitrary function of $\mathrm{y}$. Employing this expression for $\cot \alpha$ in Eq. (4.4), we obtain that the functions $\mathrm{G}(\mathrm{y})$ and $\mathrm{F}(\mathrm{y})$ must satisfy

$$
\begin{aligned}
& G^{\prime}(y) G^{\prime \prime}(y)+k^{2} \operatorname{Re} R_{H}\left[\left\{\left(\frac{F(y)}{G^{\prime}(y)}\right)^{\prime \prime}+\right.\right. \\
& \left.F(y)\left(\frac{G^{\prime \prime}(y)}{G^{\prime 2}(y)}\right)^{\prime}\right\}+\left\{\left(\frac{G^{\prime \prime}(y)}{G^{\prime 2}(y)}\right)^{\prime \prime}+\right. \\
& \left.\left.\left(\frac{G^{\prime \prime}(y)}{G^{\prime}(y)}\right)\left(\frac{G^{\prime \prime}(y)}{G^{\prime 2}(y)}\right)^{\prime}\right\}\right]=0
\end{aligned}
$$

Since this equation must hold true for all $\mathrm{x}$, the coefficients of powers of $x$ must be zero. Hence, the functions $\mathrm{G}(\mathrm{y})$ and $\mathrm{F}(\mathrm{y})$ must satisfy the two equations.

$$
\begin{aligned}
& G^{\prime}(y) G^{\prime v}(y)+k^{2} \operatorname{Re} R_{H}\left[\left\{\left(\frac{F(y)}{G^{\prime}(y)}\right)^{\prime \prime}+\right.\right. \\
& \left.F(y)\left(\frac{G^{\prime \prime}(y)}{G^{\prime 2}(y)}\right)^{\prime}\right\}=0
\end{aligned}
$$

and

$$
\left(\frac{G^{\prime \prime}(y)}{G^{\prime 2}(y)}\right)^{\prime \prime}+\left(\frac{G^{\prime \prime}(y)}{G^{\prime}(y)}\right)\left(\frac{G^{\prime \prime}(y)}{G^{\prime}(y)}\right)^{\prime}=0
$$

\section{Employing}

$$
\begin{aligned}
& \left(\frac{G^{\prime \prime}(y)}{G^{\prime 2}(y)}\right)^{\prime}=\frac{1}{G^{\prime}(y)}\left[\left(\frac{G^{\prime \prime}(y)}{G^{\prime}(y)}\right)^{\prime}-\frac{1}{G^{\prime}(y)}\left(\frac{G^{\prime \prime}(y)}{G^{\prime}(y)}\right)^{2}\right. \\
& \text { and }\left(\frac{G^{\prime \prime}(y)}{G^{\prime 2}(y)}\right)^{\prime \prime}=\frac{1}{G^{\prime}(y)}\left[\left(\frac{G^{\prime \prime}(y)}{G^{\prime}(y)}\right)^{\prime \prime}-\right. \\
& \left.3\left(\frac{G^{\prime \prime}(y)}{G^{\prime}(y)}\right)\left(\frac{G^{\prime \prime}(y)}{G^{\prime}(y)}\right)^{\prime}+\left(\frac{G^{\prime \prime}(y)}{G^{\prime}(y)}\right)^{3}\right]
\end{aligned}
$$

in Eq. (4.8), Eq. (4.8) is replaced by

$$
\left(\frac{G^{\prime \prime}(y)}{G^{\prime 2}(y)}\right)^{\prime \prime}-2\left(\frac{G^{\prime \prime}(y)}{G^{\prime}(y)}\right)\left(\frac{G^{\prime \prime}(y)}{G^{\prime}(y)}\right)^{\prime}=0
$$

For the determination of $\mathrm{G}(\mathrm{y})$ and $\mathrm{F}(\mathrm{y})$ such that $\mathrm{G}(\mathrm{y})$ satisfies (4.9), and $\mathrm{F}(\mathrm{y})$ satisfy (4.7), we have the following possible two Cases;

(1) $G^{\prime \prime}(y)=0 \operatorname{and}(2)\left(\frac{G^{\prime \prime}(y)}{G^{\prime}(y)}\right)^{\prime}=0$

\subsubsection{Case (1)}

$$
\begin{aligned}
& \text { If } G^{\prime \prime}(y)=0 \\
& \psi=G(y)=A_{1} y+B_{1}
\end{aligned}
$$

Where $\mathrm{A} 1 \neq 0$ and $\mathrm{B} 1$ are arbitrary constants.

$F(y)=A_{2} y+B_{2}$ 
Where $\mathrm{A}_{2} \neq 0$ and $\mathrm{B}_{2}$ are arbitrary constants.

Employing (4.10) and (4.11) in (3.9), (4.5), (4.2) and (3.2), we have

$$
\begin{aligned}
& \mathbf{v}=\left(A_{1}, 0\right), \alpha=\operatorname{arccot}\left(A_{2} y+B_{2}\right), \\
& \omega=0, j=\frac{-k A_{2}}{A_{1}}, \\
& H=\left(\frac{k}{A_{1}}\left(A_{2} y+B_{2}\right), \frac{k}{A_{1}}\right)
\end{aligned}
$$

We use these solutions in the linear momentum Eq. (2.12) and find e by integrating these equations. Having found e, we use (2.6) and (2.7) to find the pressure function given by

$$
\begin{aligned}
& p=e-\frac{1}{2} \rho\left(u^{2}+v^{2}\right)=\frac{k^{2} R_{H}}{A_{1}^{2}} A_{2} x- \\
& \frac{k^{2} R_{H}}{2 A_{1}^{2}}\left(A_{2} y+B_{2}\right)^{2}+\frac{A_{1}^{2}}{2}(1-\rho)
\end{aligned}
$$

\subsubsection{Case (2)}

If $\left(\frac{G^{\prime \prime}(y)}{G^{\prime}(y)}\right)^{\prime}=0$

Then $\quad \frac{\partial \psi}{\partial y}=G^{\prime}(y)=c_{2} \exp \left(c_{1} y\right)$,

$\frac{\partial \psi}{\partial x}=0$

Where $\mathrm{c} 2 \neq 0$ and $\mathrm{c}_{1}$ are two arbitrary constants. Choose $c_{1}=0$ results in case (1). Using (4.14) in (4.7), we find that $\mathrm{F}(\mathrm{y})$ satisfies the differential equation

$$
F^{\prime \prime}(y)-2 c_{1} F^{\prime}(y)=-\frac{c_{1}^{3} c_{2}^{3}}{k^{2} \operatorname{Re} R_{H}} x p\left(3 c_{1} y\right)
$$

Solving this linear equation, we have

$$
F(y)=c_{3}+c_{4} \exp \left(2 c_{1} y\right)-\frac{c_{1} c_{2}^{3}}{k^{2} \operatorname{Re} R_{H}} \exp \left(3 c_{1} y\right)
$$

Where $c_{3}$ and $c_{4}$ are arbitrary constant. Using (4.14) and (4.15) in Eqs. (3.9), (4.5), (4.2) and (3.2), we get

$$
\begin{aligned}
& \mathbf{v}=\left(c_{2} \exp \left(c_{1} y\right), 0\right) \\
& \alpha=\operatorname{arccot}\left\{c_{1} x+c_{4} \exp \left(2 x c_{1} y\right)-\right. \\
& \left.\frac{c_{1} c_{2}^{3}}{3 k \operatorname{Re} R_{H}} \exp \left(3 c_{1} y\right)+c_{3}\right\} \\
& \omega=-c_{1} c_{2} \exp \left(c_{1} y\right) \\
& j=-k\left[\frac{c_{1} x}{c_{2}} \exp \left(-c_{1} y\right)+\frac{c_{4}}{c_{2}} \exp \left(c_{1} y\right)-\right. \\
& \left.\frac{c_{1} c_{2}^{2}}{3 k \operatorname{Re} R_{H}} \exp \left(2 c_{1} y\right)+\frac{c_{3}}{c_{2}} \exp \left(-c_{1} y\right)\right] \\
& \text { and } \\
& H=k\left[\frac{c_{1} x}{c_{2}} \exp \left(-c_{1} y\right)+\frac{c_{4}}{c_{2}} \exp \left(c_{1} y\right)-\right. \\
& \left.\frac{c_{1} c_{2}^{2}}{3 k \operatorname{Re} R_{H}} \exp \left(2 c_{1} y\right)+\frac{c_{3}}{c_{2}} \exp \left(-c_{1} y\right), \frac{\exp \left(-c_{1} y\right.}{c_{2}}\right]
\end{aligned}
$$

Using (4.16) in linear momentum equations and integrating for $\mathrm{p}$, we obtain

$$
\begin{aligned}
& p(x, y)=\frac{c_{1}^{2} c_{2}}{3 \operatorname{Re}} \exp \left(-c_{1} y\right)+\frac{k R_{H}}{c_{2}}\left[\left\{\frac{c_{1} c_{4}}{c_{2}}-\right.\right. \\
& \left.\left.\frac{c_{1} c_{3}}{c_{2}} \exp \left(-2 c_{1} y\right)\right\} x-\frac{c_{1}^{2} x^{2}}{2 c_{2}} \exp \left(-2 c_{1} y\right)\right] \\
& -\frac{k R_{H}}{2 c_{2}}\left[c_{3} \exp \left(-c_{1} y\right)+c_{4} \exp \left(c_{1} y\right)-\right. \\
& \left.\frac{c_{1} c_{2}^{3}}{3 k \operatorname{Re} R_{H}} \exp \left(2 c_{1} y\right)\right]^{2}+\frac{1}{2}(1-\rho) c_{2}^{2} \exp \left(2 c_{1} y\right)
\end{aligned}
$$

\subsection{Results for Problem 1}

If the streamlines in a steady plane flow of an electrically fluid of infinite electrical conductivity are parallel straight lines and the magnetic lines are variably inclined to the streamlines in the flow plane then

(i) The possible magnetic lines pattern is of the form

$2 \mathrm{x}-\mathrm{A}_{3} \mathrm{y}^{2}-2 \mathrm{~A} 4 \mathrm{y}=$ constant

and

$$
\begin{aligned}
& 6 k \operatorname{Re} R_{H} c_{1} x+c_{1} c_{2}^{3} \exp \left(3 c_{1} y\right)+6 k \operatorname{Re} R_{H} \\
& \left(c_{3}-c_{4} \exp \left(2 c_{1} y\right)\right)=\text { constan } t
\end{aligned}
$$

(ii) The solutions to the flow problem are given by Eqs. (4.12) and (4.13) or (4.16) and (4.17) according as the magnetic lines are the family of curves (4.18) or (4.19).

Figure 1 represent the graph of the Eq. (4.18), which are magnetic lines pattern are represented by a parabolic form.

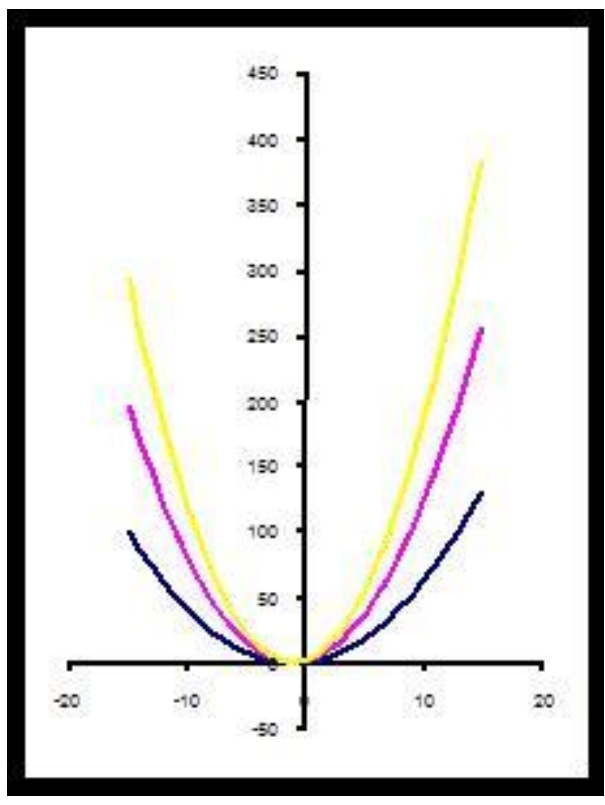

Fig.1. Magnetic line pattern of

$$
x=\frac{1+A_{1} y^{2}+2 A_{4} y}{2}
$$


C.S. Bagewadi and S. Bhagya / JAFM, Vol. 4, No. 4, pp. 77-83, 2011.

\subsection{Problem 2}

When the magnetic lines are variably inclined but nowhere aligned with the streamlines for non-viscous fluid flows. We assume

$\phi(x, y)=g(y) \quad$ and $\quad g^{\prime}(y) \neq 0$

So that $H_{1}=g^{\prime}(y) \quad$ and $\quad H_{2}=0$

Substituting (4.20) in Eqs. (4.26) and (3.27), we obtain

$$
j=-g^{\prime \prime}(y) \quad \text { and } \quad \omega=-k \frac{\partial}{\partial y}\left(\frac{\cot \alpha}{g^{\prime}(y)}\right)
$$

Employing (4.20) and (4.21) in Eqs. (3.24) and (3.25), we find that the unknown functions $\mathrm{g}(\mathrm{y})$ and $\alpha(\mathrm{x}, \mathrm{y})$ must satisfy

$$
\begin{aligned}
& \cot \alpha \frac{\partial^{2}}{\partial y \partial x}\left(\frac{\cot \alpha}{g^{\prime}(y)}\right)-\frac{\partial^{2}}{\partial y^{2}}\left(\frac{\cot \alpha}{g^{\prime}(y)}\right)=0 \\
& \text { and } \frac{\partial}{\partial x}(\cot \alpha)+\frac{g^{\prime \prime}(y)}{g^{\prime}(y)}=0
\end{aligned}
$$

solving (4.22) and (4.23), we obtain

$$
\cot \alpha=-\frac{g^{\prime \prime}(y)}{g^{\prime}(y)}+f(y)
$$

and

$$
\begin{aligned}
& {\left[\frac{1}{g^{\prime}(y)}\left(\frac{g^{\prime \prime}(y)}{g^{\prime}(y)}\right)^{\prime \prime}-\frac{2 g^{\prime \prime}(y)}{g^{\prime 2}(y)}\left(\frac{g^{\prime \prime}(y)}{g^{\prime}(y)}\right)^{\prime}\right] x-} \\
& {\left[f(y)\left(\frac{g^{\prime \prime}(y)}{g^{\prime}(y)}\right)^{\prime}+\left(\frac{f(y)}{g^{\prime}(y)}\right)^{\prime \prime}\right]=0}
\end{aligned}
$$

Where $f(y)$ is an arbitrary function of $y$. Since Eq. (4.25) must hold true for all $\mathrm{x}$, it follows that $\mathrm{g}(\mathrm{y})$ and $\mathrm{f}(\mathrm{y})$ must satisfy

$$
\begin{aligned}
& \left(\frac{g^{\prime \prime}(y)}{g^{\prime}(y)}\right)^{\prime \prime}-2\left(\frac{g^{\prime \prime}(y)}{g^{\prime}(y)}\right)\left(\frac{g^{\prime \prime}(y)}{g^{\prime}(y)}\right)^{\prime}=0 \\
& \left(\frac{f(y)}{g^{\prime}(y)}\right)^{\prime \prime}+f(y)\left(\frac{g^{\prime \prime}(y)}{g^{\prime 2}(y)}\right)^{\prime}=0
\end{aligned}
$$

There are two possible cases from (4.26) and (4.27) that is,

$$
\text { (1) } g^{\prime \prime}(y)=0 \text { and } \quad(2) \quad\left(\frac{g^{\prime \prime}(y)}{g^{\prime}(y)}\right)^{\prime}=0
$$

4.3.1 Case (1)

If $g^{\prime \prime}(y)=0$ then

$\psi=g(y)=b_{1} y+b_{2}$ and $f(y)=b_{3} y+b_{4}$

Where $b_{1}, b_{2}, b_{3}$ and $b_{4}$ are arbitrary constants. Employing (4.28) in (3.23), (4.24), (4.21) and (3.16), we have

$\mathbf{H}=\left(\mathrm{b}_{1}, 0\right), \quad \cot \alpha=b_{3} y+b_{4}$

$\omega=-\frac{k b_{3}}{b_{1}}$,

$\mathrm{j}=0$

$$
\begin{aligned}
& \mathbf{v}=\left(\frac{k}{b_{1}}\left(b_{3} y+b_{4}\right),-\frac{k}{b_{1}}\right) \text { and } \\
& p=\frac{k^{2} b_{3}}{b_{1}^{2}} x+\frac{k^{2}}{2 b_{1}^{2}}\left(b_{3} y+b_{4}\right)^{2}-\frac{k^{2} \rho}{2 b_{1}^{2}} \\
& \left(b_{3} y+b_{4}\right)-\frac{1}{2}\left[R_{H} b_{1}^{2}+\frac{k^{2} \rho}{b_{1}^{2}}\right]
\end{aligned}
$$

\subsubsection{Case (2)}

If $\left(\frac{g^{\prime \prime}(y)}{g^{\prime}(y)}\right)^{\prime}=0$

Then $\psi=g(y)=\frac{d_{2}}{d_{1}} \exp \left(d_{1} y\right)+d_{3}$

and $f(y)=d_{4}+d_{5} \exp \left(2 d_{1} y\right)$

where $\mathrm{d}_{1}, \mathrm{~d}_{2}, \mathrm{~d}_{3}, \mathrm{~d}_{4}$ and $\mathrm{d}_{5}$ are arbitrary constants. Employing (4.30) and (4.31) in (3.23), (4.24), (4.21) and (3.16), we obtain

$\mathbf{H}=\left(d_{2} \exp \left(d_{1} y\right), 0\right), \cot \alpha=d_{4}+d_{5} \exp \left(2 d_{1} y\right)-d_{1} x$

$\omega=-\frac{d_{1}^{2} k}{d_{2}} x \exp \left(-d_{1} y\right)+\frac{k}{d_{2}}$

$\left(d_{1} d_{4} \exp \left(-d_{1} y\right)+d_{1} d_{5} \exp \left(d_{1} y\right)\right.$

$j=-d_{1} d_{2} \exp \left(d_{1} y\right)$

$\mathbf{v}=\left(\frac{k}{d_{2}}\left(d_{4} \exp \left(-d_{1} y\right)+d_{5} \exp \left(d_{1} y\right)-\right.\right.$

$\left.\left.d_{1} x \exp \left(-d_{1} y\right)\right),-\frac{k}{d_{2}} \exp \left(-d_{1} y\right)\right)$

$p(x, y)=\frac{k d_{1}^{2}}{2 d_{2}^{2}}\left[\exp \left(-2 d_{1} y\right)\right] x^{2}+\frac{k^{2} d_{1}}{d_{2}^{2} \exp \left(d_{1} y\right)}$

$\left[d_{5} \exp \left(d_{1} y\right)-d_{2} d_{4} \exp \left(-d_{1} y\right)\right]$

$+\frac{k^{2}}{2 d_{2}}\left[d_{4} \exp \left(-d_{1} y\right)+d_{5} \exp \left(d_{1} y\right)\right]^{2}-$

$\frac{d_{2}^{2}}{2} R_{H} \exp \left(2 d_{1} y\right)-\frac{k^{2} \rho}{2 d_{2}}\left[\left(d_{4} \exp \left(-d_{1} y\right)\right.\right.$

$\left.\left.+d_{5} \exp \left(d_{1} y\right)-d_{1} x \exp \left(-d_{1} y\right)\right)^{2}+\exp \left(-2 d_{1} y\right)\right]$

\subsection{Results for Problem 2}

If the magnetic lines in a steady plane flow of an electrically conducting non viscous fluid of infinite electrically conductivity are straight lines parallel to $\mathrm{x}$ axis and the streamlines are variably inclined to the magnetic lines in the flow plane, then

(i) The possible streamline pattern is of the form

$2 x+b_{3} y^{2}+2 b_{4} y=$ Constant

and

$2 d_{1} x-2 d_{1}\left(d_{1}-d_{4}\right) y+d_{5} \exp \left(2 d_{1} y\right)=$ Constant $(4.34)$

(ii) The solution to the flow problem are given by the Eqs. (4.29) or (4.32) according to the streamlines (4.33) or (4.34) respectively. 
Figure 2 represent the graph of the Eq. (4.33), which is streamlining pattern represented by a parabolic form. Figure 3 represent the graph of the Eq. (4.34), which is streamline pattern.

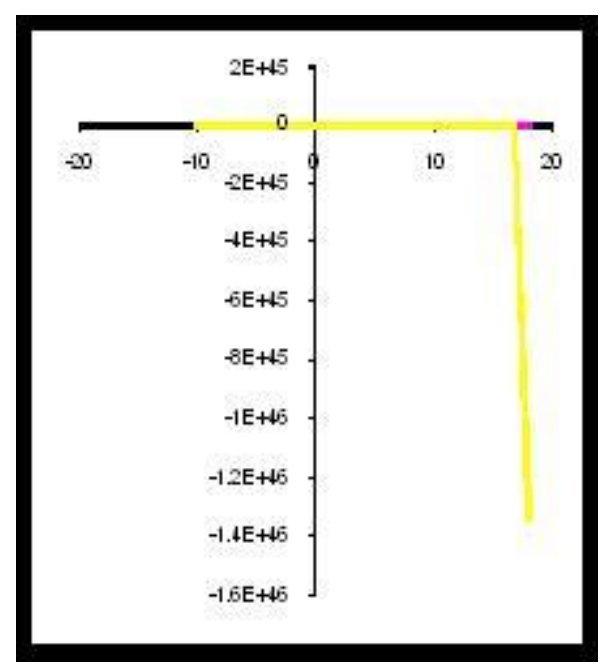

Fig. 2. Streamline pattern of

$$
x=\frac{1+2\left(d_{1}-d_{4}\right) y-d_{5} \exp \left(2 d_{1} y\right)}{2 d_{1}}
$$

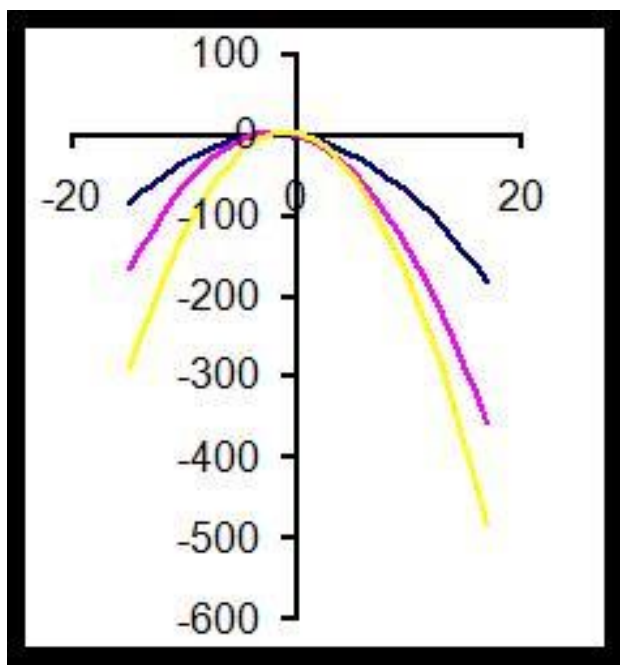

Fig. 3. Streamline pattern of

$$
x=\frac{1-b_{3} y^{2}-2 b_{4} y}{2}
$$

\section{CONCLUSION}

MHD flows when the velocity and magnetic fields are variably inclined are analyzed. In problem 1 , when the magnetic lines are variably inclined but nowhere aligned with the streamlines and the magnetic lines are constantly inclined. In problem 2 , If the magnetic lines in a steady plane flow of an electrically conducting non viscous fluid of infinite electrical conductivity are straight lines parallel to $\mathrm{x}$-axis and the streamlines are variably inclined to the magnetic lines in the flow plane. The parabolic nature of streamlines and magnetic lines are presented in the graphs.

\section{REFERENCES}

Anjali Devi, S.P. and B. Ganga (2010). Dissipation effects on MHD non-linear flow and heat transfer past a porous surface with prescribed heat flux. $J$. Appl. Fluid. Mech 3 (1), 1-6.

Bagewadi, C.S. and S. Bhagya (2004). Behaviour of Streamlines in aligned flow. Far East J. Appl. Math.17(2),121-138.

Bagewadi, C.S. and S. Bhagya (2006). A study of streamlines in second grade fluid Flows. Map. J. Sci. 5(1), 5-20.

Bagewadi, C.S. and S. Bhagya (2007). Solutions of second grade fluids flow. Bull. Cal. Math. Soc. 99 (5), 485-500.

Bagewadi, C.S. and Siddabasappa (1993). The plane rotating viscous MHD flows. Bull. Cal. Math. Soc. $85,513-520$.

Bagewadi, C.S. and Siddabasappa (1995). Stady plane rotating MHD flows with contant velocity magnitude along each steamline. J. Shivaji Univ. (Science) 31, 137-143.

Chandna, O.P. and M.R. Garg (1979). On steady plane magnetohydrodynamic flows with orthogonal magnetic and velocity fields. Int. J. Engng. Sci. 17, 251-257.

Chandna, O.P., R.M. Barron and K.T. Chew (1982). Hodograph transformations and solutions in variably inclined MHD plane flows. J. Engg. Math. 16, 223-243.

Chandna, O.P., R.M. Barron and K.T. Chew (1989). Finitely conducting orthogonal magneto hydrodynamic plane flows. Can. J. Phy. 67, 56-62.

Chandna, O.P. and P.V. Nguyen (1990). Hodograph transformation method and solutions in aligned MHD plane flows. Int. J. Engng. Sci. 28(10), 973987.

Martin, M.H. (1971). Flow of viscous fluid. I. Arch. Rational Mech. and Analysis 41,266-286.

Rahmati, A.R. and M. Ashrafizaadeh (2009). A Generalized Lattice Boltzmann Method for ThreeDimensional Incompressible Fluid Flow Simulation. J. Appl. Fluid. Mech. 2(1), 71-95. 